\title{
A Micro Force Measurement and Tracing System Based on Dead-Weight-Balanced Hinge
}

\author{
Zhiyi Wang $^{1, \mathrm{a}}$, Meirong Zhao ${ }^{1, \mathrm{~b}}$, Peiyuan Sun ${ }^{1, \mathrm{c}}$, Yaqian Han ${ }^{1, \mathrm{~d}}$ and Yelong Zheng ${ }^{1, \mathrm{e}, *}$ \\ ${ }^{1}$ State Key Laboratory of Precision Measuring Technology and Instruments, Tianjin University, \\ Tianjin 300072, China. \\ azhiyi_wang@qq.com, bmeirongzhao@tju.edu.cn, csun_peiyuan@tju.edu.cn, d450908247@qq.com, \\ ezhengyelongby@163.com
}

Keywords: Micro force, Dead-weight-balanced hinge, Stiffness, Resolution.

\begin{abstract}
In micro force measurement system, transfer mechanism can transform micro force into traceable standard quantity and export them. It is the key link of force measurement. Transfer mechanism is not directly involved in force calculation, but determines measurement capability of system. Because traditional parallelogram hinge is easy to produce plastic deformation after a long time hanging, and has large creep, we design a flexible hinge mechanism whose dead weight is balanced. This mechanism reduces creep of hinge, and enhances measurement accuracy. Initial deformation of hinge has been decreased and resolution has been increased. Experiment demonstrates that resolution of this system is up to $10^{-8} \mathrm{~N}$, and relative standard uncertainty of force is less than $2 \%$.
\end{abstract}

\section{Introduction}

At present, micro/nano force has been widely used in new materials, biological medicine, microelectronics, aerospace, national defense, and many other areas, but there is not an internationally unified measurement method for force less than $10^{-5} \mathrm{~N}$ yet [1,2]. In order to promote key technology of related field, various countries are studying micro force measurement method.

Force transmission and transformation play an important role in micro force measurement system based on electrostatic field. Especially in $10^{-6} \mathrm{~N}$ force measurement system, force transmission mechanism is required to have high sensitivity and repeatability accuracy, appropriate limits on degree of freedom and well dynamic characteristics. Because of zero friction, small volume, no clearance and smooth movement, flexible hinge mechanism catches people's fancy. A lot of micro force measurement systems, such as electrostatic force balance of NIST [3,4], nano-force calibrator of KRISS [5] and force calibration system of Center for Measurement Standards in Taiwan [6-8], choose flexible hinge as force transfer mechanism. But hinges' structure of NIST and KRISS are complex, and have large stiffness. And that of CMS is always in a suspended state for a long time, leading to plastic deformation of hinge, which is bad for a long-time use.

In this paper, based on micro force measurement device in electrostatic field, we put forward and design a hinge mechanism which can balance out dead weight of itself. Moreover, in order to measure force in the range of $10^{-6} \mathrm{~N} \mathrm{to} 10^{-4} \mathrm{~N}$, we optimize structure design upon traditional hinge. 


\section{Structure and Principle of Micro Force Measurement System}

Structure of micro force measurement system is shown as Fig.1.

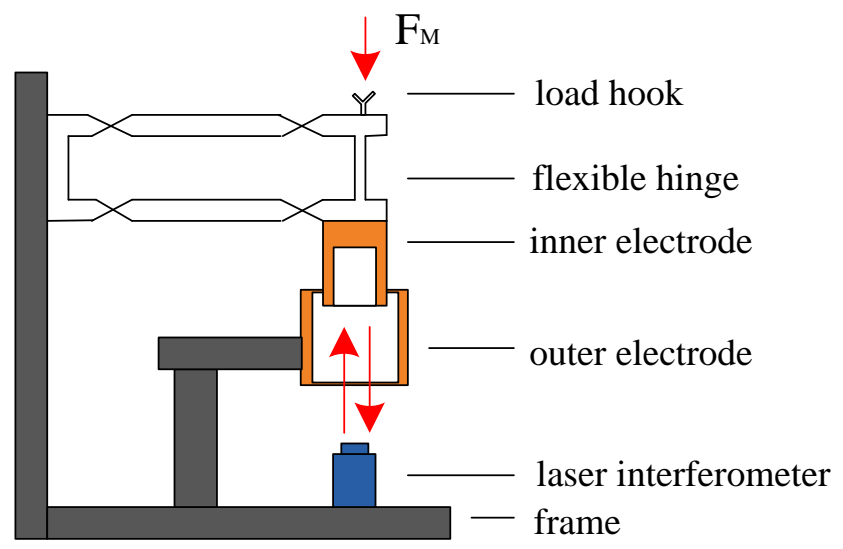

Fig.1 Diagram of system structure

This system is based on principle of electrostatic field. We use variable area coaxial cylindrical capacitance sensor with inner electrode fixed on flexible hinge and outer electrode fixed on the frame. When micro force to be measured is applied on load hook, inner electrode will move with flexible hinge in the vertical direction. Displacement of inner electrode can be detected by laser interferometer in the bottom. According to principle of electrostatic force $[9,10]$, when the voltage is applied on inner and outer electrode, force along axial line will act on both of them. The relationship between electrostatic force and voltage is shown as Eq. (1):

$$
F_{e}=\frac{1}{2} \frac{d C}{d z} U^{2}
$$

In Eq. (1), $U$ is voltage difference between inner and outer electrode, $d C / d z$ is the capacitance gradient, where $d z$ is relative displacement of electrodes, $d C$ is capacitance change resulting from $d z$ and can be measured by high precision capacitance bridge.

Under the action of electrostatic force, inner electrode will move down. By adjusting the voltage $\mathrm{U}$, inner electrode can keep in the equilibrium position. Recording the voltage value, and putting it into Eq. (1), we can get electrostatic force which equals to micro force to be measured. So that the measurement and traceability of micro force can be realized.

\section{Design of Dead-Weight-Balanced Hinge}

\subsection{Structure of Dead-Weight-Balanced Hinge}

Because inner electrode is hung on the flexible hinge, and fixed way of hinge determines it subjects to its dead weight, there is always a vertical down load on the thin wall of hinge, leading to its stress and deformation, which are main results of creep. So we design a dead-weight-balanced flexible hinge mechanism, shown as Fig.2. Because the dead weight of hinge has been balanced, deformation on thin wall of hinge is smaller. This mechanism decreases the stress and initial deformation of hinge, and reduce its equivalent stiffness. This mechanism is a parallelogram mechanism consisted of four horizontal single hinges and five vertical single hinges. We add a lever on traditional parallelogram hinge, so that the hinge can keep horizontal by adjusting counter poise on the end of lever. Now dead weight of flexible hinge is balanced by lever and counter poise. 


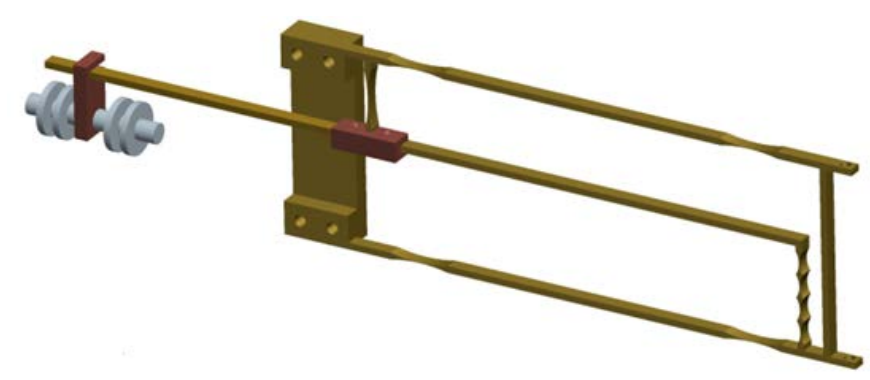

Fig.2 Diagram of dead-weight-balanced hinge

\subsection{Stiffness of Dead-Weight-Balanced Hinge}

We use finite element method to simulate and analyze the structure of hinge. put a downward force on the end of hinge, and its deformation is shown in Fig.3. Vertical displacement in point A is $5.026 \mathrm{~mm}$, and horizontal displacement is $0.001 \mathrm{~mm}$; Vertical displacement in point B is $5.1 \mathrm{~mm}$, and horizontal displacement is $0.37 \mathrm{~mm}$. So, compared with vertical displacement, horizontal displacement can be ignored. That is to say, five vertical single hinges can be regarded as strings, and do not affect stiffness of whole structure. So we only need to calculate stiffness of parallelogram hinge and central lever.

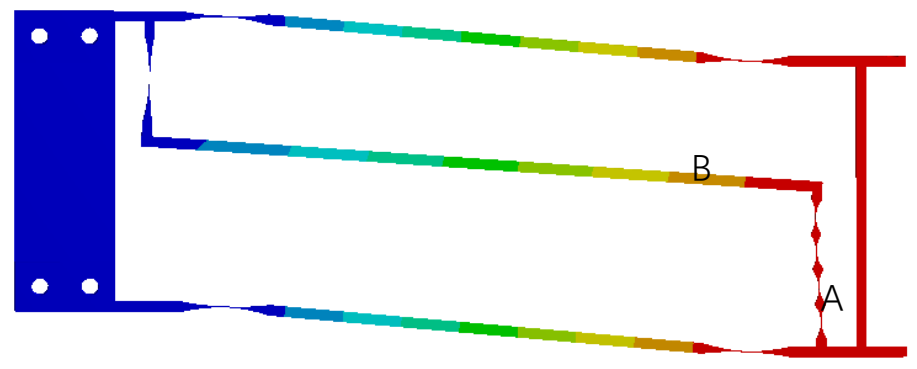

Fig.3 Diagram of hinge’s deformation

Stiffness of parallelogram hinge has been analyzed, now we calculate stiffness of central lever. Force analysis of central lever is shown as Fig.4.

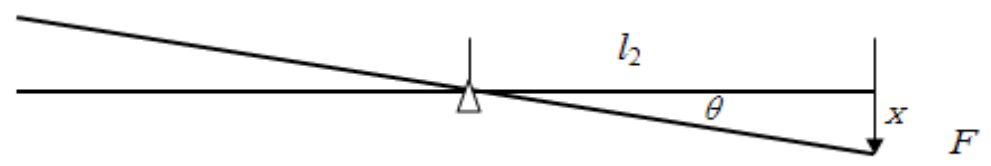

Fig.4 Force analysis diagram of central lever

Under the effect of $F$, the end of lever moves down. The torsional rigidity of lever is:

$$
k_{\theta}=\frac{E b r^{3}}{12 R f\left(\frac{r}{t}\right)}
$$

Because of:

$$
k_{\theta}=\frac{T}{\theta}=\frac{F l_{2}}{x / l_{2}}=\frac{F l_{2}^{2}}{x}=k_{\text {lever }} l_{2}^{2}
$$

Then stiffness of central lever is:

$$
k_{\text {lever }}=\frac{k_{\theta}}{l_{2}^{2}}
$$

According to geometrical relationship, angle deformation quantity $\theta$ of five notches is equal. Combining with Eq. (4), we can get stiffness calculation formula of dead-weight-balanced hinge: 


$$
k=k_{\text {lever }}+k_{\text {hinge }}=k_{\theta}\left(\frac{1}{l_{2}^{2}}+\frac{4}{l^{2}}\right)=\frac{E b r^{3}}{12 R f\left(\frac{r}{t}\right)}\left(\frac{1}{l_{2}^{2}}+\frac{4}{l^{2}}\right)
$$

where

$$
f(x)=\frac{12 x^{4}(2 x+1)}{(4 x+1)^{5 / 2}} \times \tan ^{-1} \sqrt{4 x+1}+\frac{2 x^{3}\left(16 x^{2}+4 x+1\right)}{(4 x+1)^{2}(2 x+1)}
$$

$E$ is elasticity modulus of beryllium bronze, $t$ is thickness of hinge's notches, $b$ is width of hinge, $R$ is major axis radius and $r$ is minor axis radius of notch ellipse, $l$ is effective length of hinge and $l_{2}$ is effective length of lever arm.

We set size of hinge as: $t=0.1 \mathrm{~mm}, b=4 \mathrm{~mm}$. $R=15 \mathrm{~mm}, r=5 \mathrm{~mm}, l=104 \mathrm{~mm}, l_{2}=138 \mathrm{~mm}$, and according to Eq. (5), the theoretical stiffness of dead-weight-balanced hinge is $6.9951 \mathrm{~N} / \mathrm{m}$.

\subsection{Creep Property of Dead-Weight-Balanced Hinge}

A creep test is conducted on dead-weight-balanced hinge, and result is shown in Fig.5.

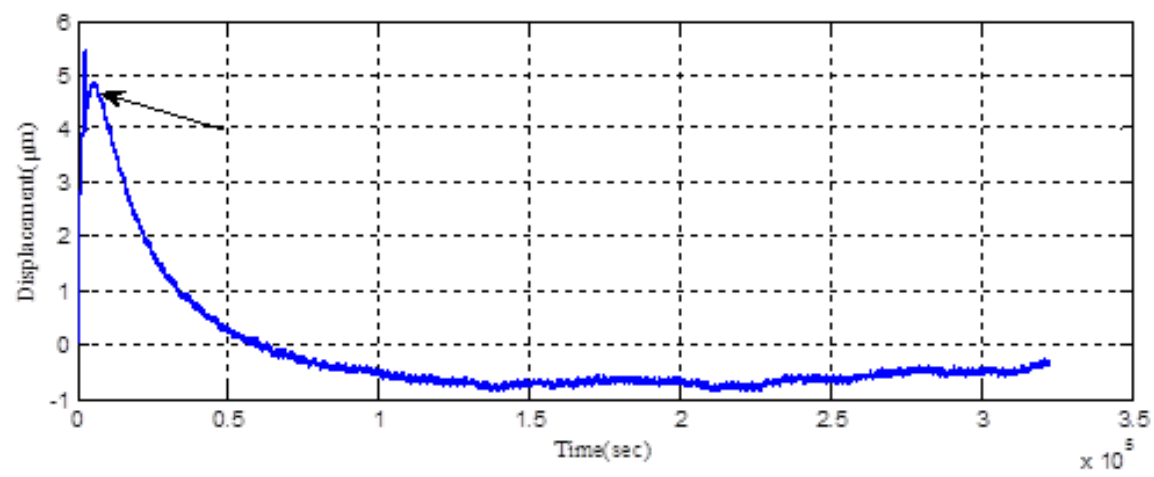

Fig.5 Creep test result of dead-weight-balanced hinge

We can see that creep is less than $1 \mu \mathrm{m}$ in 72 hours. This structure increases the stability of micro force measurement system.

\subsection{Spectrum Analysis of Dead-Weight-Balanced Hinge}

In the measurement process, one end of dead-weight-balanced hinge is fixed on the fixed base, the other end is connected to inner electrode. This structure makes that hinge occurs resonance oscillation easily under the effect of external vibration. Through the spectrum analysis of the vibration of hinge, resonant frequency can be find, and we can choose appropriate vibration isolation method to reduce the influence of external vibration on the measurement.

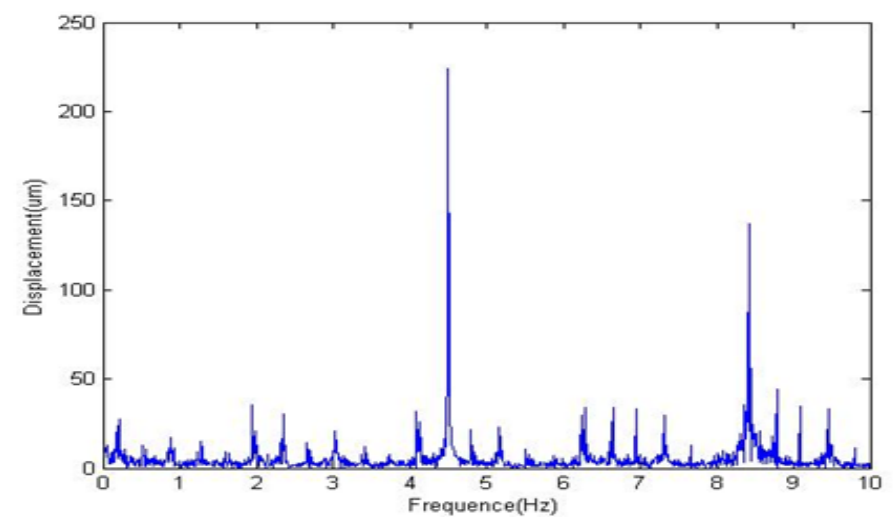

Fig.6 Step response spectrum analysis of dead-weight-balanced hinge

From Fig.6 we can see that resonant frequency of dead-weight-balanced hinge $F_{q}$ is $4.5 \mathrm{~Hz}$. So external vibration whose frequency is around $F_{q}$ have a great influence on the hinge. 


\section{System Experiment of Dead-Weight-Balanced Hinge}

\subsection{System Resolution Experiment}

Resolution of micro force measurement system is mainly limited by external environment disturbance and self-accuracy. To test resolution of micro force measurement system, in no-load condition, inner electrode keeps in equilibrium under the control of electrostatic force, and output voltage is recorded. In this way, environmental noise, control accuracy and structure accuracy are all considered.

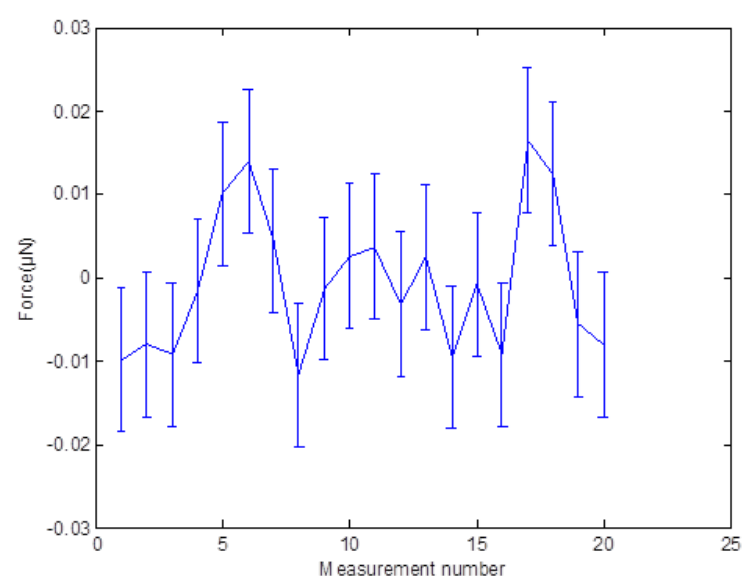

Fig.7 Result of system resolution experiment

Result of system resolution experiment is shown as Fig.7, and linear creep has been wiped off. Standard deviation of 20 measurement results is $9 \mathrm{nN}$. So resolution of micro force measurement system is up to $10^{-8} \mathrm{~N}$.

\subsection{Comparison Between Electrostatic Force and Mass}

In order to measure accuracy of micro force measurement system, electrostatic force is compared with metal weights which are calibrated by mass comparator. Gravitational acceleration $g$ is $9.8011 \mathrm{~N} / \mathrm{kg}$. Use our measurement system to measure gravity of gold foil loop, and compare with result of mass comparator. Comparison result is shown in Table 1.

Table 1 comparison between electrostatic force and mass

\begin{tabular}{ccccccc}
\hline No. & $\begin{array}{c}\text { Result of mass } \\
\text { comparator } G \\
(\mu \mathrm{N})\end{array}$ & $\begin{array}{c}\text { Electrostatic } \\
\text { force } F_{\mathrm{e}} \\
(\mu \mathrm{N})\end{array}$ & $\begin{array}{c}\text { Standard } \\
\text { variance } \\
(\mu \mathrm{N})\end{array}$ & $\begin{array}{c}\text { Relative } \\
\text { variance } \\
(\%)\end{array}$ & $\begin{array}{c}G-F_{\mathrm{e}} \\
(\mu \mathrm{N})\end{array}$ & $\begin{array}{c}\left(G-F_{\mathrm{e}}\right) / G \\
(\%)\end{array}$ \\
\hline 1 & 55.24 & 55.7 & 0.5 & 0.9 & -0.46 & -0.83 \\
2 & 75.62 & 76.7 & 0.6 & 0.8 & -1.08 & -1.42 \\
3 & 115.36 & 118.3 & 0.5 & 0.4 & -2.94 & -2.54 \\
\hline
\end{tabular}

\subsection{System Uncertainty Analysis}

Measure standard mass for several times, and their variance is type A uncertainty, including uncertainty caused by ground vibration and air disturbance. And uncertainty caused by voltage, capacitance gradient and laser interferometer is type B uncertainty. Uncertainty of micro force measurement system is compounded by these two type uncertainty, as shown in Table 2. 
Table 2 uncertainty of micro force measurement system

\begin{tabular}{cc}
\hline Source of uncertainty & Proportion (\%) \\
\hline Repetitive error & 1 \\
Capacitance gradient & 0.08 \\
Voltage & $\sqrt{\frac{4.1}{430 f^{3}}}$ \\
Laser interferometer & $6 / f$ \\
\hline Compound uncertainty & $\sqrt{1+0.0064+\frac{36}{f^{2}}+\frac{4.1}{430 f^{3}}}$ \\
\hline
\end{tabular}

From Table 2, we can see that if force to be measured is $10 \mu \mathrm{N}$, uncertainty caused by voltage is so small that can be ignored, uncertainty caused by laser interferometer is $0.6 \%$, and uncertainty caused by repetitive error is $1 \%$. So compound uncertainty of system is less than $2 \%$.

\section{Conclusions}

In this study, we apply dead-weight-balanced hinge into micro force measurement system to overcome plastic deformation of traditional parallelogram hinge because of hanging for a long time. Stiffness of system has been reduced through decreasing pre-deformation of hinge. Resolution of system is up to $10^{-8} \mathrm{~N}$ and relative standard uncertainty is less than $2 \%$. This system has large measurement range and high accuracy, and lays a good foundation for measurement of smaller force.

\section{Acknowledgements}

This work was financially supported by National Natural Science Foundation of China (No.61304246) and National Key Technology Research and Development Program of the Ministry of Science and Technology of China (No. 2011BAK15B06).

\section{References}

[1] Pratt J R, Kramar J A, Newell D B, et al. Review of SI traceable force metrology for instrumented indentation and atomic force microscopy[J]. Measurement science and technology, 2005, 16(11): 2129.

[2] Nesterov V. Facility and methods for the measurement of micro and nano forces in the range below $10^{-5} \mathrm{~N}$ with a resolution of $10^{-12} \mathrm{~N}$ (development concept) [J]. Measurement Science and Technology, 2007, 18(2): 360.

[3] Pratt J R, Kramar J A. SI realization of small forces using an electrostatic force balance[C] // Proc. XVIII IMEKO World Congress on Metrology for a Sustainable Development (Rio de Janeiro, Brazil, 17-22 September 2006). 2006, 109.

[4] Kramar J A, Newell D B, Pratt J R. NIST electrostatic force balance experiment[J]. VDI BERICHTE, 2002, 1685: 71-76.

[5] Kim M S, Pratt J R, Brand U, et al. Report on the first international comparison of small force facilities: a pilot study at the micronewton level[J]. Metrologia, 2011, 49(1): 70.

[6] Chen S J, Pan S S. A force measurement system based on an electrostatic sensing and actuating technique for calibrating force in a micronewton range with a resolution of nanonewton scale[J]. Measurement Science and Technology, 2011, 22(4): 045104. 
[7] Chen S J, Pan S S. Nanonewton force generation and detection based on a sensitive torsion pendulum[J]. IEEE Transactions on Instrumentation and Measurement, 2009, 58(4): 897-901.

[8] Chen S J, Pan S S, Yeh Y S, et al. Measurement of cantilever spring constant using an electrostatic sensing and actuating force measurement system[J]. Measurement Science and Technology, 2014, 25(11): 115006.

[9] Pratt J R, Newell D B, Kramar J A, et al. Probe-force calibration experiments using the NIST electrostatic force balance[C]//Proceedings of the American Society for Precision Engineering 2003 Winter Topical Meeting. 2003: 64-69.

[10] Qi Y, Wang H, Lin Y, et al. Measuring system for micro force based on electrostatic theory[C]//Electronic Measurement \& Instruments, 2009. ICEMI'09. 9th International Conference on. IEEE, 2009: 1-524-1-527. 